\title{
Violence urbaine, démocratie et changement culturel : l'expérience brésilienne (Partie 3)
}

\section{Angelina Peralva}

\section{(2) OpenEdition}

1 Journals

\section{Édition électronique}

URL : http://journals.openedition.org/conflits/708

DOI : $10.4000 /$ conflits.708

ISSN : $1777-5345$

Éditeur :

CCLS - Centre d'études sur les conflits lilberté et sécurité, L'Harmattan

Édition imprimée

Date de publication : 15 octobre 1998

ISSN : 1157-996X

Référence électronique

Angelina Peralva, «Violence urbaine, démocratie et changement culturel : l'expérience brésilienne (Partie 3) », Cultures \& Conflits [En ligne], 29-30 | automne-hiver 1998, mis en ligne le 16 mars 2006, consulté le 30 mars 2021. URL : http://journals.openedition.org/conflits/708 ; DOI : https://doi.org/ $10.4000 /$ conflits.708

Ce document a été généré automatiquement le 30 mars 2021.

Creative Commons License 


\title{
Violence urbaine, démocratie et changement culturel : l'expérience brésilienne (Partie 3)
}

\author{
Angelina Peralva
}

1 Le lynchage

2 Il est difficile d'établir avec exactitude le nombre de cas de lynchage survenus au Brésil depuis deux décennies. Pour une évaluation approximative, on ne peut que se reporter aux enquêtes existantes, c'est-à-dire à des banques de données établies à partir de revues de presse.

3 La première étude (Benevides, 1982) a établi un recensement de 75 cas pour la période 1979-1981, dont 32 avec des victimes fatales et 43 tentatives de lynchage ${ }^{1}$. José de Souza Martins se réfère à un total de 272 cas, dont 262 concernant la période 1979-1988; plus 4 cas isolés, entre 1970 et 197841 . De cet ensemble, 145 (53,3\%) correspondraient à des cas ayant eu comme issue la mort de la victime. Ce recensement a été établi à partir de deux quotidiens de São Paulo. J. S. Martins ne cache pas l'incomplétude de son univers de référence : en comparant ses cas à ceux de l'étude de $\mathrm{M}$. V. Benevides, il lui apparaitt que l'élargissement de la revue de presse à des quotidiens d'autres régions du pays aurait mis en évidence un nombre bien plus grand de faits de ce type. O. Hanashiro, J. Sinhoretto et H. Singer, travaillant à partir de plusieurs journaux de São Paulo et Rio de Janeiro dans le cadre d'une recherche menée par le Centre d'études de la violence, concernant la période 1980-1989, et d'une recherche menée par la Commission Teotonio Vilela pour la période 1991-1993, ont établi un recensement de 442 cas, dont $70 \%$ environ avec des issues fatales ${ }^{2}$.

4 J. S. Martins indique que l'accroissement du phénomène du lynchage coïncide avec la redémocratisation: " Les données, apparemment, ne suggèrent pas l'idée d'un continuum, avec un accroissement progressif du nombre de lynchages. Néanmoins, si nous séparons les dix dernières années (jusqu'à 1984 et à partir de 1985³), une information en ressort clairement : pendant les quatre années qui se situent entre 1985 et 1988 , c'est-à-dire depuis le début du nouveau régime politique, celui de la 'Nouvelle 
République', 136 cas (de lynchage) ont eu lieu ; alors que pendant les quatre dernières années du régime militaire, entre 1981 et 1984, 91 cas ont eu lieu. Cela veut dire que le nombre de lynchages a été de presque $50 \%$ plus important dans la nouvelle situation politique, c'est dire, une fois et demi le nombre de lynchages et de tentatives de lynchages survenus lors de la période finale de la dictature ". Et il ajoute: " Accroissement similaire à celui qui a eu lieu en milieu rural, où la violence est devenue plus intense maintenant qu'auparavant "4.

5 J. S. Martins souligne par ailleurs la portée sociologique générale du phénomène, au sens où les auteurs des lynchages ne sont pas seulement issus des strates populaires ; on retrouve également des lyncheurs issus des couches moyennes. Il l'inscrit dans un moment historique défini, autant en termes politiques que sociaux, par la liminarité : " Je pense, particulièrement, à ces personnes situées sur le 'fil du rasoir', dans cet espace de transition inachevée, et d'indéfinition, qui comprend la périphérie des villes et les zones rurales, où les rapports de propriété, de travail et d'autorité sont en train de se dissoudre. J'entends qu'il est possible de définir l'espace et le temps où survient ce type de violence, qui est au seuil de l'urbain, mais aussi au seuil de la politique "5. Elles n'ont par contre recensé que dix cas survenus dans l'Etat de Pará, nombre inférieur à celui établi par J. S. Martins ${ }^{6}$.

6 En ce qui concerne le mobile à l'origine du lynchage, J. S. Martins distingue quatre catégories : " 1. violations de principes sociaux de coexistence et de réciprocité $(8,7 \%)$; 2. crimes contre la personne - tels que le viol, l'agression, le meurtre $(43,1 \%) ; 3$. crimes contre la personne et la propriété - tel le fait de tuer pour voler $(13,4 \%) ; 4$. crimes contre la propriété - cambriolages, vols, etc. $(32,4 \%)$. Cependant, il note que les cas de vols et de cambriolages qui suscitent des lynchages ont comme victimes, très souvent, des travailleurs pauvres; et il en déduit que, là aussi, il faut le comprendre comme un crime contre la personne, contre la survie du travailleur et de sa famille. " Il ne s'agit pas d'un crime contre l'avoir, mais contre l'être "7.

7 M. V. Benevides distingue deux grandes catégories de lynchages: anomiques et communautaires $^{8}$. Dans les lynchages anomiques, les auteurs sont des passants qui se constituent en tant que collectif autour d'une action ponctuelle qu'ils identifient comme une agression commise contre quelqu'un. Les erreurs de jugement, dans ce type de lynchage, ne sont pas exclues et des innocents peuvent en être les victimes ${ }^{9}$. Dans les lynchages qu'on peut définir comme communautaires, la vengeance préméditée est plus explicitement à l'oeuvre dans la dynamique de la violence.

8 Les données d' $\mathrm{O}$. Hanashiro, J. Sinhoretto et H. Singer permettent une estimation de l'importance relative de ces deux différentes logiques. D'abord, elles montrent que la plupart des lynchages organisés ont eu à leur origine des crimes contre la personne (33 cas sur 39), alors que les lynchages occasionnels ont eu comme mobile un crime contre le patrimoine (17 cas sur 28) ${ }^{10}$. Deuxièmement, en ce qui concerne les auteurs des lynchages, la même étude montre que, dans $45,4 \%$ des cas étudiés, il s'agissait de gens de passage et dans $1,4 \%$ des cas, d'usagers des transports en commun, dans $1,6 \%$ des cas, de détenus partageant une même cellule - ainsi dans $48,4 \%$ des cas étudiés, il s'agissait d'un ensemble de personnes réunies par le hasard des circonstances. D'un autre côté, dans 30,5\% des cas étudiés, les lyncheurs appartenaient à un groupe de voisinage, dans $6,2 \%$ ils étaient des habitués du lieu où est survenu le lynchage, dans $4,3 \%$ des cas ils étaient des proches ou appartenaient à la famille de la personne agressée par celui qui deviendra ensuite la victime d'un lynchage - c'est dire que dans 
$41 \%$ des cas, des bases de constitution d'une action communautaire étaient préalablement données. Il faut enfin considérer le cas des corporations professionnelles, d'où sont issus les auteurs de $10,5 \%$ des cas de lynchage pris en compte dans l'étude - dont celui d'une corporation à part, les chauffeurs de taxi (souvent victimes d'agressions et d'attaques à main armée), responsables à eux seuls de $8,1 \%$ des cas. C'est dire que $10,5 \%$ des lynchages viennent d'une communauté constituée sous la forme d'une corporation professionnelle. Si on y ajoute le premier type d'action communautaire, on constatera que les lynchages communautaires correspondent à $51,5 \%$ de l'ensemble des cas de lynchages examinés dans l'étude, contre $48,4 \%$ de lynchages pratiqués par des groupes occasionnels.

En ce sens, Martins (1989: 25) attire l'attention sur l'importance des rites à l'oeuvre dans certains lynchages. " J'ai enregistré des cas où la capture et l'exécution de la victime ont été effectuées de façon clairement ritualisée et où les participants ont fait preuve d'une grande sérénité. Dans l'un de ces cas, survenu dans un quartier de São Paulo, un habitant, responsable de nombreux délits commis à répétition à l'encontre de ses voisins, a été soumis au jugement d'une sorte de tribunal populaire : un jour, dans la matinée, des gens qui arrivaient à une boulangerie du coin, pour acheter leur pain et leur lait, ont été conviés à rester et à prendre une décision concernant ce délinquant. Après délibération, un groupe est allé le chercher chez lui pour le conduire dans cet établissement commercial. Sur place, il a entendu l'accusation, on lui a donné la parole, on lui a demandé s'il voulait qu'on appelle sa famille pour pouvoir faire ses adieux, on lui a offert une dernière cigarette, on l'a amené dans la rue et on l'a tué par des jets de pierre et des coups de bâton. " Un autre cas du même genre est survenu dans la région métropolitaine de Rio de Janeiro. " Le corps de la victime est resté sur place pendant plusieurs jours, jeté comme un déchet dans la rue. Tous les jours les habitants se rassemblaient en silence devant ce cadavre, jusqu'à ce que la police en soit enfin informée. " Le lynchage s'effectue pratiquement toujours dans la rue, ou dans un lieu public, jamais dans un espace privé. " Il semblerait que se cache derrière cette démarche l'idée que le lynchage n'est pas un crime, justement parce qu'il a lieu dans un endroit public, sous la forme d'une action collective. Le crime, c'est ce qu'on pratique en cachette, à l'abri du regard d'autrui. Pour cette raison le lynchage est public, pratiqué à la vue et avec la complicité, volontaire ou non, de tous. " (Martins, 1989 : 27) Les punitions sont très rares. Une enquête sur l'absence de prise en charge par la justice du phénomène du lynchage est en cours au Centre d'études de la violence de l'Université de São Paulo.

Le vigilantisme ${ }^{11}$

11 Contrairement au lynchage, expression de la réaction directe d'un groupe à une agression prétendument subie, le vigilantisme relève de différentes logiques de semiprofessionnalisation d'une police parallèle - on les désigne comme " les justiciers " ou " les tueurs ", à São Paulo, " les exterminateurs " à Rio de Janeiro où, inversement, il s'inscrit dans le dépassement des frontières de la légalité par des vigiles employés dans des entreprises de sécurité privée. Dans tous les cas, il est un indicateur supplémentaire de l'ampleur de l'espace à travers lequel la privatisation de la sécurité se déploie au Brésil.

Il n'y a quasiment pas d'enquêtes disponibles sur le premier type de vigilantisme. Il faut signaler l'étude réalisée par Fernandes (1992) sur la base d'un nombre très limité d'entretiens biographiques avec des " justiciers " incarcérés, et avec leur famille. 
L'étude met en évidence la centralité, dans la justification des meurtres perpétrés, d'un jugement moral à l'égard de la victime - jugement partagé par la famille du tueur, et axé sur une opposition entre la norme et la déviance, entre les travailleurs et les criminels. Autrement dit, autant chez les tueurs que chez leur famille, le travail semble être une valeur centrale dans le processus de constitution des représentations légitimatrices de ces pratiques.

13 L'enquête menée par un journaliste de O Dia, quotidien populaire de Rio de Janeiro, à propos des " exterminateurs " de la Baixada Fluminense, et publiée à travers une série d'articles (Oliveira, 1993), va également dans le sens de la centralité des catégories morales dans la légitimation de leurs pratiques. Cependant la logique morale n'est pas la seule à intervenir; l'argent est tout de même au coeur de l'expérience de " l'extermination ". Ces actes se développent en symbiose avec le commerce local. Dans la région métropolitaine de Rio de Janeiro, certains " exterminateurs " seraient devenus de riches propriétaires de voitures, de résidences secondaires et même d'un centre commercial. En ce sens, ils sont à la frontière des pratiques illégales des agents des entreprises de sécurité privée étudiés par Caldeira (1992).

Selon cette dernière, " il y a un consensus parmi les hommes d'affaires, dans le domaine de la sécurité privée, quant au fait que les contrôles (exercés par le gouvernement sur ce type de service) sont moindres qu'auparavant (du temps du régime militaire) " (Caldeira,1992 : 198). Par ailleurs, on estime à soixante-dix le nombre d'entreprises de sécurité privée illégales en fonctionnement dans l'Etat de São Paulo. " La plupart des gens mêlés à ce marché marginal sont soit des policiers, soit des ex-policiers, qui ne peuvent pas être enregistrés comme des agents de la sécurité privée. En général, ils utilisent des armes de la police et travaillent les jours où ils ne sont pas de service, profitant de leur emploi du temps ( 48 heures de service en alternance avec 48 heures de repos). Naturellement, ce marché ne comporte pas de registre. On emploie aussi des armes du marché noir, bien plus puissantes que celles dont l'usage est autorisé pour les agents enregistrés, et même pour la police. Quelques-unes de ces entreprises, récemment fermées par la police fédérale, avaient à leur tête des ex-policiers mêlés à l'escadron de la mort, ou des justiciers bien connus (...). " (Caldeira, 1992 :202). Les services de sécurité employés par des résidences fermées sont assurés par ces entreprises illégales. D'un côté, les services des entreprises légales sont trop chers; de l'autre, embaucher ses propres gardiens est, réglementairement, excessivement compliqué. " De grandes résidences fermées disposent, et de services avec des agents armés, et de services avec des agents non armés (...) ; et la plupart des services armés sont assurés par des entreprises illégales. " De telles entreprises se situent à la frontière de ce qu'on désigne comme le phénomène des justiciers. " L'un des aspects les plus sérieux du marché clandestin de la sécurité, c'est sa proximité avec les escadrons de la mort qui agissent dans la région métropolitaine de São Paulo. Souvent, la façade d'une entreprise de sécurité privée, c'est tout ce dont ont besoin les justiciers pour faire leur travail. Par ailleurs, les justiciers sont parfois le seul type de sécurité privée dont disposent les gens pauvres. Trouvant que la police régulière n'est pas à même de les protéger, et incapables de se payer d'autres services de sécurité privée, beaucoup de gens, spécialement des commerçants dans la périphérie, se retournent vers ceux dont ils pensent qu'ils ont le pouvoir de les protéger, et dont ils peuvent rémunérer les services. " (Caldeira, 1992 : 203).

Les meurtres d'enfants et d'adolescents 

faiblesse de la démocratie brésilienne. Plus de quarante-cinq mille adolescents, entre quinze et dix-neuf ans ont été assassinés entre 1979 et 1994 (Toledo, 1996). Ce phénomène, complexe, est assez significatif des tensions d'une société incapable d'assurer son propre ordre intérieur, et qui en même temps développe une perception des enfants et des jeunes comme des fauteurs de désordre à éliminer, faute de pouvoir les soumettre. Il est vrai que cette fracture, générationnelle, se recoupe d'une autre, sociale. Si les jeunes - et toutes les statistiques le confirment - sont les victimes préférentielles de pratiquement toutes les modalités de violence urbaine, les enfants, les adolescents et la jeunesse pauvre, en général, plus particulièrement celle qui mène dans la rue une partie de son existence, voire toute son existence, le sont (et de loin) davantage.

17 Si la violence à l'encontre des jeunes jaillit plus souvent dans les milieux populaires, elle est loin de s'astreindre à ces strates-là et elle correspond à une brutalité bien plus générale qu'on retrouve dans tous les milieux. Un meurtre illustre particulièrement bien notre propos. En décembre 1983, Joilson de Jesus, un garçon des rues de quinze ans qui était suivi par les agents de la Pastorale ${ }^{12}$ de l'enfance, a été tué en pleine journée dans le centre-ville de São Paulo. Ayant volé une chaîne en or au cou d'une passante ${ }^{13}$, il a été attrapé par un homme qui l'a d'abord subjugué et qui ensuite, en le piétinant, l'a tué. Cet homme était un Procureur de la justice de l'Etat de São Paulo. Ce crime n'a jamais été pénalement condamné. est le massacre de Candelaria, une église du centre ville de Rio de Janeiro à proximité de laquelle, en juillet 1993, huit enfants des rues ont été mitraillés dans la nuit par des policiers militaires et des ex-policiers militaires. Les meurtriers ont été reconnus par la seule victime qui a survécu à ses blessures et qui vit actuellement en Suisse. Leur jugement, une première - il est très rare que les enquêtes concernant ces événements aboutissent et que leurs auteurs soient jugés - a débuté en 1996. Une première condamnation a eu lieu.

19 De tels meurtres ont lieu un peu partout dans le pays, dans les zones urbaines de tous les Etats de la fédération. Ils ont été à l'origine de la formation d'un mouvement, dans le domaine du militantisme des droits de l'homme, le Mouvement National des Garçons et des Filles des rues - MNMMR, en fait constitué surtout par des éducateurs de rue, lesquels, par divers moyens, ont cherché à doter ces enfants d'une capacité à résister à la violence. Ce mouvement a sans doute contribué à une meilleure compréhension de l'expérience de la rue et de la violence qui lui est attachée, mais surtout à une transformation profonde (quoique encore inachevée) des modalités de prise en charge de cette population fragile que sont les enfants des rues.

20 La littérature disponible distingue deux catégories d'enfants, dans l'expérience de la rue - ceux qui travaillent dans la rue mais qui gardent des liens réguliers avec leur milieu familial ; et ceux qui vivent en rupture familiale et en faisant de la rue leur seul lieu de survie. Les frontières entre ces deux mondes sont fragiles. Ils se croisent en permanence et l'une des sorties de la situation de travail dans la rue peut être la rupture familiale et l'installation radicale dans l'expérience de vie dans la rue. La distinction analytique entre les deux catégories a permis néanmoins de montrer que la population des enfants vivant dans les rues était infiniment moins nombreuse que celle des enfants travaillant dans les rues. A Rio de Janeiro, des organisations non 
gouvernementales ont quadrillé la ville et effectué des comptages simultanés, à des heures tardives, des enfants dormant dans les rues. (Fase, Ibase, Idac, Iser, 1992). Elles ont abouti à une estimation de l'ordre d'un peu plus de cinq cents enfants supposés vivre de façon permanente dans les rues.

Une recherche ultérieure (Soares, Milito et Silva, 1996) a permis d'inverser la perception de la rue comme un lieu globalement menaçant, mettant systématiquement en péril la vie des enfants. Portant sur les enquêtes policières engagées sur des meurtres d'enfants à Rio de Janeiro, ses résultats ont mis en évidence l'opposition spatiale entre les lieux où ces meurtres sont commis et ceux où se concentrent les enfants vivant dans les rues. Dès lors, il a été possible de comprendre que des événements comme ceux de Candelaria, avec des enfants tués dans le centre de la ville, étaient somme toute rares. Les agressions à leur encontre interviennent plus souvent à proximité de leur lieu de résidence, dans des quartiers populaires de la région métropolitaine, que dans les quartiers riches vers lesquels ils se déplacent et où ils finissent par s'installer en permanence. L'enquête a aussi permis de comprendre que l'une des principales raisons pour lesquelles des adolescents, et même de très jeunes enfants, se réfugiaient dans les rues, loin de leur domicile, était les menaces de mort (émanant de " justiciers " ou d'exterminateurs ") qui pesaient sur eux. Des situations de désagrégation familiale peuvent aussi être à l'origine de leur départ, mais il n'est pas certain que de telles raisons soient les plus fréquentes.

Suite à la Constitution de 1988, toute la politique concernant les droits des enfants mineurs a été révisée et a abouti à la promulgation de la Loi n 8069 du 13 juillet 1990, dite ECA - et portant sur le Statut de l'enfant et de l'adolescent. En assurant à tous les enfants au-dessus de douze ans le droit de libre circulation à l'intérieur du territoire national, et en soumettant les enfants pouvant être perçus comme en situation de risque aux orientations de Conseils Tutélaires, constitués dans chaque commune par des conseillers élus au sein de la société civile, les auteurs de la loi ont, d'un côté, institué les enfants comme des sujets de droit; de l'autre, ils les ont soustraits, au moins en partie, aux risques de la violence policière. Contrairement à ce qui se passait avant, il est aujourd'hui illégal de conduire un enfant des rues dans un commissariat de police. Des structures spéciales existent désormais pour les accueillir ${ }^{14}$.

Mais par ailleurs, le droit de libre circulation, assuré à tous les enfants au-dessus de douze ans, outre le fait qu'il crée une tension et une ambiguïté au niveau du rapport parents-enfants et de l'autorité parentale (ce droit est suspendu en cas de délit jugé et en tout état de cause, jamais pour une période supérieure à trois ans), a induit une refonte profonde dans les conceptions de prise en charge des enfants des rues. Plus exactement, le renouvellement de ces conceptions, fondé sur de nouvelles pratiques des éducateurs de rue, lancées dès le début des années quatre-vingt, précède la nouvelle forme légale. Le principe de l'enfermement dans des institutions, dont les enfants en situation de risque et sous la tutelle de l'Etat faisaient habituellement l'objet, était une politique qui a prévalu pendant une large période. Désormais, au contraire, on prend ces enfants en charge à partir de la situation de rue, en acceptant de les resocialiser progressivement et de transformer la sortie de la rue non pas en une contrainte pour eux, mais en un choix personnel, tout en assurant les conditions matérielles susceptibles de rendre un tel choix possible. De nombreuses expériences en ce sens se sont développées sur tout le territoire national ${ }^{15}$. 
24 De récentes mettent en évidence les risques relatifs de l'une et de l'autre de ces politiques de prise en charge, l'ancienne et la nouvelle. La première (Silva, 1996) analyse une cohorte de trois cent soixante-dix enfants orphelins et abandonnés qui, sous le régime militaire (période pendant laquelle les conditions de prise en charge de ces enfants se sont considérablement durcies), ont vécu en institution depuis leur plus tendre enfance. Silva a pu montrer qu'au moins un tiers d'entre eux s'étaient engagés dans une carrière criminelle, avec des taux de récidive de l'ordre de $70 \%$.

Maria Tedrus (1996) étudie une expérience à São Bernardo do Campo, une ville industrielle de la région métropolitaine de São $\mathrm{Paulo}^{16}$. Les premières tentatives de prise en charge des enfants des rues à São Bernardo avaient été développées, dans les années quatre-vingt, par de jeunes étudiants d'une université locale, en coopération avec diverses organisations. Elles avaient eu alors une issue dramatique, avec l'assassinat de plusieurs de ces enfants par un groupe de justiciers. Le choc causé par cet événement, l'un des rares où l'identification et la punition des coupables ont été possibles, a favorisé l'instauration d'une nouvelle situation dans le centre ville de São Bernardo, la sécurité des enfants qui y travaillent, en gardant des voitures stationnées ou en rendant de menus services aux passants, étant désormais mieux assurée. Dès lors, l'étude de Maria Tedrus montre l'équilibre instable, mais réel, qui définit l'expérience de ces enfants travaillant dans la rue, entre des liens familiaux qui se maintiennent, une éducation scolaire difficile à préserver, mais toujours valorisée, et cette partie de la journée passée dans la rue, non pas seuls, mais au sein d'un réseau d'entraide formé par d'autres enfants dans la même situation, réseau que l'auteur de l'étude appelle une confrérie.

Quoique le risque de dérive, de rupture et de marginalisation soit toujours présent, ce que l'étude montre est au-delà. Alors que dans l'expérience de la galère, étudiée en France par François Dubet, dominent la désorganisation, l'exclusion et la rage, dans la confrérie au contraire dominent l'organisation, l'inclusion et l'autonomie individuelle ${ }^{17}$. La famille, d'un côté, et la confrérie, de l'autre, opèrent comme des médiations complémentaires (qui se légitiment réciproquement par ailleurs) pour la construction d'une relation autonome entre le sujet et le monde, à travers une combinaison possible entre autonomie et protection. La famille fonctionne comme un espace régulé de contrôle, de protection et de solidarité, fondé sur une complicité affective, ce qui constitue l'expression organisée d'une expérience sociale. La capacité de l'enfant à mobiliser des ressources personnelles, lui permettant de s'insérer dans un marché, s'inscrit dans une logique d'inclusion dans la société et de participation à la consommation (Maria Tedrus a d'ailleurs montré que seule une partie infime et symbolique des revenus de l'enfant est remise à la famille). La confrérie, enfin, constitue l'élément de médiation qui assure les conditions subjectives lui permettant d'affronter les aléas d'un travail somme toute doté d'une faible légitimité mais qui, par le peu d'argent qu'il procure à l'enfant, lui assure une autonomie individuelle extrêmement valorisée et qui contribue au processus de construction de sa personnalité. La confrérie resocialise le risque toujours présent dans cette expérience, qui cesse dès lors d'être purement individuelle. Sans idéaliser l'expérience du travail enfantin dans la rue, et tout en faisant état des fortes tensions qui lui sont inhérentes, Tedrus montre que les nouvelles modalités de prise en charge de ces enfants sont somme toute plus intéressantes et plus positives que celles auxquelles a été soumise la génération d'enfants institutionnalisés étudiés par Silva. 

domaines les plus variés; elles ont des implications précises du point de vue de la définition même des problèmes sociaux et des réponses à y apporter; et surtout elles peuvent être elles-mêmes source de violence, puisqu'elles bouleversent d'anciennes formes de régulation des liens sociaux et que dans le vide que ne viennent guère combler de nouvelles formes de régulation, elles créent un espace favorable au déploiement de la violence. L'exemple des enfants des rues illustre ce phénomène. Les enfants travaillent dans la rue, dit Maria Tedrus, non pas parce que leur contribution en matière de revenus est essentielle à la survie familiale, mais parce que ces revenus leur assurent une possibilité d'inclusion en tant que consommateurs personnels dans la société de masse ${ }^{19}$. Ceci renvoie, il est certain, à la faiblesse des revenus familiaux, qui ne sont pas en eux-mêmes suffisants pour leur assurer cette inclusion, mais aussi à une nouvelle définition des besoins individuels en matière de consommation. C'est dans les limites de cette tension que la valorisation de l'autonomie individuelle de l'enfant induit les familles à accepter le risque que représente leur travail dans la rue - un risque dont les familles sont bien conscientes, dit Tedrus, qui les a longuement interrogées.

31 Le côté le plus anomique de cette expérience, celui où l'enfant se consacre à de menus vols et où il devient rapidement une victime potentielle des justiciers, payés par des commerçants excédés, en constitue naturellement le versant sombre. Mais il indique a contrario deux grandes tendances qu'il faut prendre en compte dans la formulation des politiques publiques de sécurité: d'abord, les nouvelles limites dans lesquelles 
s'inscrivent les régulations propres à l'univers familial, la famille devenant davantage un milieu affectif essentiel à la construction de la personnalité de l'enfant qu'un lieu de contrainte pour lui, et de régulation normative. Et ensuite, la présence de l'enfance dans la rue ayant tendance à devenir irréversible, se pose la question de la nécessité de nouvelles formes de régulation de l'espace public, susceptibles de compléter utilement l'action familiale et d'assurer convenablement la protection de l'enfant. Les grandes lignes de ces politiques sont indiquées dans l'expérience analysée par Tedrus - d'un côté, la présence d'éducateurs de rue auprès des enfants établit une médiation entre eux et les adultes; de l'autre, la mise à l'écart des modalités les plus dramatiques de la violence qui les menace, celle des justiciers, leur permet d'y évoluer dans une relative sécurité. La violence contre les enfants des rues, qui persiste dans la société brésilienne, relève de tout ce qui échappe à ces nouvelles formes de régulation émergentes.

un espace dont le sens n'est pas nécessairement cohérent avec celui qui se développ sur le plan des relations familiales et des rapports parents-enfants. On observe, par rapport au modèle classique de la socialisation, une désynchronisation entre le sens de l'expérience familiale et la forte demande d'ordre qu'on retrouve dans la société au sens large. Ce décalage est particulièrement sensible dans le discours de certains enseignants, qui en viennent à demander une rééducation des familles, pour que les enfants leur arrivent plus disciplinés et avec des comportements jugés plus adéquats 
aux conditions de fonctionnement du travail scolaire (Peralva et Chamlian, 1984). Dès lors, il faut dire que la forte demande d'ordre qu'on constate dans la société brésilienne (et qui ne se solde pas nécessairement par la violence) est sociale au sens large, mais s'inscrit en faux contre l'évolution des moeurs observée au sein des familles.

La disponibilité à la violence est commune aux deux générations qui s'opposent, mais elle n'a pas, pour chacune d'entre elles, la même signification. Les gens au-dessus de quarante ans expriment le plus directement une demande d'ordre et, en même temps, la nostalgie des régulations sociales qui étaient propres au monde du travail: la valorisation de l'effort, de l'épargne, et de la gratification différée - des pratiques qui contenaient en elles-mêmes tout un univers de valeurs et de symboles aujourd'hui en voie de disparition. Evidemment, des jeunes (et notamment de jeunes policiers) peuvent adhérer à de telles valeurs et participer à la logique de crispation qui s'y révèle autour d'un modèle d'ordre en voie de décomposition. Dans l'enquête de Paulo Oliveira et son équipe sur les exterminateurs de la Baixada Fluminense, il a été constaté que cette police parallèle était formée par des gens autour de la quarantaine ${ }^{21}$. Rappelons par ailleurs, l'enquête de Heloisa Fernandes, qui met en évidence la centralité du travail dans l'univers des valeurs des justiciers de São Paulo et de leurs familles ${ }^{22}$.

Les jeunes, victimes préférentielles de la violence et eux-mêmes souvent mêlés à des pratiques violentes, sont perçus comme des fauteurs de désordre et constituent notamment la cible de la justice illégale et de la violence policière. S'ils pratiquent euxmêmes la violence, celle-ci n'est pas de même nature que celle de leurs aînés. Même leur rapport au travail se construit autrement. Il n'est pas une valeur en soi, ni déterminé par une nécessité de survie stricto sensu; il se subordonne à un désir de consommation et d'autonomie individuelle, qui souvent conduit le jeune à une occupation esthétisée de la scène urbaine, avec la formation de groupes faiblement structurés autour d'un look ou d'une pratique musicale, et l'entraine dans différentes logiques d'affirmation de soi. La violence est une des formes possibles de mise en œuvre de cette expérience générale.

Revenir à la partie précédente

\section{NOTES}

1. Benevides Maria Victoria, "Linchamentos : violência e 'justiça' popular " in Violência Brasileira, São Paulo, Brasiliense, 1982.

2. Plus exactement, les auteurs indiquent que dans $30 \%$ des cas survenus au cours de la période 1980-1989, l'intervention de la police avait permis de sauver la victime (Hanashiro Olaya, Sinhoretto Jacqueline e Singer Helena, " Linchamentos : a democracia mudou alguma coisa ? ", dans Direitos Humanos no Brasil, n², São Paulo, NEV-USP e CTV, 1995, p. 78.)

3. 1985 est l'année où prend ses fonctions José Sarney, premier président civil depuis 1984, indirectement élu vice-président de la République par le Congrès national, et 
ayant succédé à Tancredo Neves en raison du décès de ce dernier, survenu avant même sa prise de fonctions.

4. Cf. Ibidem.

5. . Or justement, de son analyse, il ressort que les Etats où les lynchages ont été plus fréquents, au cours de la période considérée, sont São Paulo (131 cas), Rio de Janeiro (47 cas), Bahia ( 32 cas) et Pará ( 15 cas). Cette constatation n'est pas démentie dans l'étude de 0 . Hanashiro, J. Sinhoretto et $\mathrm{H}$. Singer où l'Etat de São Paulo figure avec 188 cas $(50,8 \%)$, Rio de Janeiro avec 78 cas $(21,1 \%)$ et Bahia avec 52 cas $(14,1 \%)$ [ Hanashiro Olaya, Sinhoretto Jacqueline e Singer Helena, op. cit., p. 84.

6. Il convient de noter que la banque de données établie par ce dernier inclut des cas qui lui ont été communiqués par la Pastorale de la Terre, et qui n'ont pas été diffusés dans la presse.

7. Ibidem, p. 24.

8. Benevides Maria Victoria, "Linchamentos : violência e 'justiça' popular " in Violência Brasileira, São Paulo, Brasiliense, 1982.

9. Cependant, le nombre de victimes de lynchages (pour lesquelles l'existence d'un casier judiciaire pourrait constituer un indice de culpabilité) est assez significatif dans les données relevées par Hanashiro O., Sinhoretto J., Singer H. (op. cit., p. 80) : ce nombre s'élève à 126 sur un total de 519 victimes (certains lynchages faisant plus d'une victime), c'est-à-dire, $24,3 \%$ de l'ensemble des victimes ; mais $97 \%$ des cas (130) pour lesquels on dispose d'informations. Ce qui définit le lynchage, non pas comme l'expression d'une violence aveugle, mais d'une violence communautaire (on pourrait aussi dire comme un outil de reconstruction symbolique d'une communauté imaginaire en voie de dissolution), ce que suggère d'ailleurs Martins (1989).

10. Hanashiro O., Sinhoretto J., Singer H., op. cit., p. 77-78.

11. Cette expression, empruntée de l'expérience américaine, a été surtout employée par Pinheiro (1992)

12. Les pastorales sont des organisations souples regroupant des religieux et des laïcs autour des secteurs progressistes de l'église catholique et qui interviennent sur plusieurs fronts sociaux : les problèmes agraires, raciaux, ou ceux liés à l'enfance, parmi d'autres.

13. On trouve une quantité innombrable de marchands d'or dans les rues du centre ville de São Paulo et l'or ramené par un enfant représente pour lui de l'argent comptant.

14. J'ai visité deux de ces structures. Le Commissariat de protection à l'enfance et à l'adolescence (DPCA), à Rio de Janeiro, se trouve dans un lieu isolé de la ville. Le policier qui m'y a accompagné, en voiture, m'a raconté que lorsque des enfants y sont amenés pour la première fois ils ont très peur. Ils craignent qu'on les y conduise pour les tuer. A São Paulo, la structure d'accueil s'appelle SOS Enfant. C'est un lieu très apprécié par les enfants des rues. Un fonctionnaire de la Compagnie Paulista des Transports Municipaux, qui gère le réseau des trains urbains dans la ville, après avoir menacé un gamin pris en flagrant délit, lorsqu'il pratiquait le surf du rail, de le conduire au SOS Enfant, s'est entendu dire par ce dernier : " C'est très bien là-bas ! " Il est très rare qu'une institution au Brésil soit appréciée de ses usagers.

15. Un tel bouleversement n'a été naturellement possible que parce qu'il s'est effectué dans une conjoncture marquée par l'effondrement des politiques publiques, et par la montée en force de diverses modalités de prise en charge directement par la société civile, à travers des organisations non gouvernementales souvent financées par des 
institutions étrangères. Pendant un moment, l'opposition entre les orientations publiques pour l'enfance et celles émanant des organisations non gouvernementales a été claire ; par la suite cependant, les secondes ont, au moins en partie, infléchi les premières.

16. Tedrus Maria Aparecida Lealdini, Jovens : trabalho nas ruas e experiências de sociabilidade, Dissertaçao de mestrado, São Paulo, Faculdade de Educaçao, Universidade de São Paulo, 1996.

17. Dubet François, La Galère. Jeunes en survie, Paris, Gallimard, 1987.

18. Dans le travail du groupe Axé (expression d'origine africaine signifiant approximativement "salut"), la musique, la danse et la référence à la culture noire en général prennent une place importante dans le travail éducatif développé auprès des enfants des rues.

19. Tedrus Maria Aparecida Lealdini, op. cit

20. Tedrus Maria Aparecida Lealdini, op. cit.

21. Oliveira Paulo et als, " Série de reportagens sobre os exterminadores da Baixada Fluminense ", O Dia, 4, 5, 6, 7, 9, 10, 11 et 19 juillet 1993.

22. Fernandes Heloisa Rodrigues, " Violência e Modos de vida : 'Os Justiceiros' ", Tempo Social, Revista de Sociologia, USP, São Paulo 4 (1-2) :43-52, 1992.

INDEX

Index géographique : Amérique du Sud, Brésil

Mots-clés : villes/ sociologie urbaine, violence, démocratie 